SHOCK, Vol. xx, No. x, pp. 1-9, 2017

\title{
DURATION OF UNTREATED CARDIAC ARREST AND CLINICAL RELEVANCE OF ANIMAL EXPERIMENTS: THE RELATIONSHIP BETWEEN THE "NO-FLOW" DURATION AND THE SEVERITY OF POSTCARDIAC ARREST SYNDROME IN A PORCINE MODEL
}

AQ1

\author{
Gioanni Babini, ${ }^{\star \dagger}$ Luigi Grassi,, ${ }^{\dagger}$ Ilaria Russo, ${ }^{\star}$ Deborah Novelli, ${ }^{*}$ \\ Antonio Boccardo, ${ }^{\ddagger}$ Anita Luciani, ${ }^{*}$ Francesca Fumagalli, ${ }^{*}$ Lidia Staszewsky, \\ Fabio Fiordaliso, ${ }^{*}$ Marcella De Maglie, ${ }^{\neq \S}$ Monica Salio, ${ }^{*}$ Davide D. Zani, ${ }^{\neq}$ \\ Teresa Letizia," Serge Masson, ${ }^{*}$ Mario V. Luini," Davide Pravettoni, ${ }^{\prime}$ \\ Eugenio Scanziani, ${ }^{\ddagger \S}$ Roberto Latini," and Giuseppe Ristagno* \\ *IRCCS-Istituto di Ricerche Farmacologiche "Mario Negri," Milan, Italy; ${ }^{\dagger}$ Dipartimento di Fisiopatologia \\ Medico-Chirurgica e dei Trapianti, University of Milan, Italy; ${ }^{\ddagger}$ Dipartimento di Medicina Veterinaria, \\ University of Milan, Italy; ${ }^{\S}$ Mouse and Animal Pathology Lab (MAPLab), Fondazione Filarete, Milan, Italy; \\ "Endocrininology Laboratory, Luigi Sacco Hospital, Milan, Italy; and"Istituto Zooprofilattico della \\ Lombardia e dell'Emilia Romagna - Sezione di Lodi, Lodi, Italy
}

Received 24 Apr 2017; first review completed 25 May 2017; accepted in final form 25 May 2017

\begin{abstract}
Introduction: The study investigated the effect of untreated cardiac arrest (CA), that is, "no-flow" time, on postresuscitation myocardial and neurological injury, and survival in a pig model to identify an optimal duration that adequately reflects the most frequent clinical scenario. Methods: An established model of myocardial infarction followed by $\mathrm{CA}$ and cardiopulmonary resuscitation was used. Twenty-two pigs were subjected to three no-flow durations: short (8-10 $\mathrm{min})$, intermediate $(12-13 \mathrm{~min})$, and long $(14-15 \mathrm{~min})$. Left ventricular ejection fraction (LVEF) was assessed together with thermodilution cardiac output (CO) and high sensitivity cardiac troponin T (hs-cTnT). Neurological impairment was evaluated by neurological scores, serum neuron specific enolase (NSE), and histopathology. Results: More than $60 \%$ of animals survived when the duration of CA was $\leq 13 \mathrm{~min}$, compared to only $20 \%$ for a duration $\geq 14 \mathrm{~min}$. Neuronal degeneration and neurological scores showed a trend toward a worse recovery for longer no-flow durations. No animals achieved a good neurological recovery for a no-flow $\geq 14 \mathrm{~min}$, in comparison to a $56 \%$ for a duration $\leq 13 \mathrm{~min}(P=0.043)$. Serum NSE levels significantly correlated with the no-flow duration $(r=0.892)$. Longer durations of CA were characterized by lower LVEF and CO compared to shorter durations $(P<0.05)$. The longer was the no-flow time, the higher was the number of defibrillations delivered $(P=0.043)$. The defibrillations delivered significantly correlated with LVEF and plasma hsCTnT. Conclusions: Longer no-flow durations caused greater postresuscitation myocardial and neurological dysfunction and reduced survival. An untreated CA of $12-13 \mathrm{~min}$ may be an optimal choice for a clinically relevant model.
\end{abstract}

KEYWORDS_Animal model, cardiac arrest, no-flow, outcome, postcardiac arrest syndrome

\section{INTRODUCTION}

Cardiac arrest (CA) is burdened by high mortality and severe neurological impairment among survivors (1). Up to $64 \%$ of out-of-hospital $(\mathrm{OH}) \mathrm{CA}$ victims die on the scene or on the way to the hospital during ambulance transport (2). Moreover, more than half of the successfully resuscitated patients die within $72 \mathrm{~h}$ after hospital admission, such that final survival is approximately $8 \%$ in Europe, with great differences among countries (2-4). Patients who eventually survive to hospital discharge frequently suffer from a persistent neurocognitive impairment that deeply impacts the quality of life $(1,4,5)$. Postcardiac arrest syndrome (PCAS) is responsible for these subsequent inhospital mortality and poor neurological outcome (3).

Address reprint requests to Giuseppe Ristagno, MD, IRCCS-Istituto di Ricerche Farmacologiche "Mario Negri," Via La Masa 19, 20156 Milan, Italy.

AQ3 E-mail: gristag@gmail.com

The abstract of this paper was accepted for presentation at the SMART Symposium 2016, Milan, Italy and GB was the recipient of the "Best Abstract" award

The authors report no conflicts of interest.

The study was supported in part by:the Italian Ministry of Health, Ricerca Finalizzata, bando "Giovani Ricercatori," grant no. n.46/GR-2011-02348099, to AQ4 GR; and by Fondazione Sestini, Bergamo, Italy, to GR.

DOI: 10.1097/SHK.0000000000000914

Copyright (C) 2017 by the Shock Society
Novel therapeutic approaches have been conceived and tested to improve outcome of CA. Indeed, experimental models represent the starting point for evaluating the effect of new interventions before their implementation into clinical practice. However, CA models are often not consistent in methods and experimental designs, such that their findings might be hardly reproduced in humans. An example is represented by the duration of untreated CA, known as "no-flow" time, that, although recognized as one of the main determinants of the PCAS severity, remains highly variable in the experimental studies (6-11). This potentially raises concerns on the adequacy of available experimental models in reflecting the real clinical scenario (12).

Clinical studies report an average emergency medical service arrival time to the CA scene of 6-8 min; however, considering the intervals for emergency call, ambulance dispatch, arrival to the patient, and onset of cardiopulmonary resuscitation (CPR), it is reasonable that the no-flow duration accounts for even longer periods. Based on that, the duration of untreated CA in experimental models should reasonably last more than $8 \mathrm{~min}$ to adequately reflect the clinical OHCA environment (4,13-16). Indeed, a shorter or a longer experimental no-flow duration may be associated with a lesser or a 
more severe PCAS in the animal, respectively, such that the studied interventions may overestimate or fail to show any effect on outcome.

The main focus of the present study is to provide evidence for designing more clinically relevant animal models, able to resemble CA outcomes similar to those reported in humans more recently, that is, a long-term survival with favorable neurological recovery in approximately $50 \%$ of resuscitated patients $(4,6)$. Thus, the study systematically investigates the effect of different durations of untreated CA on postresuscitation myocardial and neurological injury and survival in a pig model of CPR with the aim to identify an optimal no-flow interval to be used for interventional experimental studies.

\section{PATIENTS AND METHODS}

All procedures involving animals and their care were in conformity with national and international laws and policies. Approval of the study was obtained by the institutional review board committee and governmental institution. To systematically investigate the effect on outcome of different durations of untreated $\mathrm{CA}$, the present study includes data from an earlier investigation in which animals were subjected to $8 \mathrm{~min}$ of untreated CA (8), and data from an immediately subsequent investigation directed to increase the duration of noflow up to a clinical relevant model (in term of survival and neurological recovery), in which animals were prospectively subjected to a duration of noflow progressively increased from 10 to $12,13,14$, and 15 min. Investigators and methods of induction of CA, of CPR, and measurements were the same in all the experiments.

\section{Animal preparation}

Twenty-two male domestic pigs $(40 \pm 1 \mathrm{~kg})$ were fasted the night before experiment except for free water access. Anesthesia was induced by intramuscular injection of ketamine $(20 \mathrm{mg} / \mathrm{kg})$ followed by intravenous administration of propofol $(2 \mathrm{mg} / \mathrm{kg})$ and sufentanyl $(0.3 \mu \mathrm{g} / \mathrm{kg})$ through an ear vein access. Anesthesia was then maintained by continuous intravenous infusion of propofol $(4-8 \mathrm{mg} / \mathrm{kg} / \mathrm{h})$ and sufentanyl $(0.3 \mu \mathrm{g} / \mathrm{kg} / \mathrm{h})$. A cuffed tracheal tube was placed, and animals were mechanically ventilated with a tidal volume of $15 \mathrm{~mL} / \mathrm{kg}$ and $\mathrm{FiO}_{2}$ of 0.21 . Respiratory frequency was adjusted to maintain the end-tidal partial pressure of carbon dioxide $\left(\mathrm{EtCO}_{2}\right)$ between 35 and 40 $\mathrm{mmHg}$, monitored with an infrared capnometer (8). For measurement of aortic pressure, a fluid-filled 7F catheter was advanced from the right femoral artery into the thoracic aorta. For measurements of right atrial pressure (RAP), core temperature, and cardiac output $(\mathrm{CO})$, a $7 \mathrm{~F}$ pentalumen thermodilution catheter was advanced from the right femoral vein into the pulmonary artery. Conventional pressure transducers were used (MedexTransStar, Monsey, NY) Myocardial infarction was induced in a closed-chest preparation by intraluminal occlusion of the left anterior descending (LAD) coronary artery (8). More in details, a $6 \mathrm{~F}$ balloon-tipped catheter was inserted from the right common carotid artery and advanced into the aorta and then into the LAD, beyond the first diagonal branch, with the aid of image intensification and confirmed by injection of radiographic contrast media. For inducing ventricular fibrillation (VF), a 5F pacing catheter was advanced from the right jugular vein into the right ventricle. The position of all catheters was confirmed by characteristic pressure morphology and/or fluoroscopy. Frontal plane electrocardiogram was recorded. Heart rate and rhythms were monitored by electrocardiograms.

\section{Experimental procedure}

The balloon of the LAD coronary artery catheter was then inflated with $0.7 \mathrm{~mL}$ of air to occlude the flow. Occlusion was confirmed by the rapid occurrence of progressive electrocardiographic ST segment elevation. If VF did not occur spontaneously, after $10 \mathrm{~min}$ it was induced with 1-2 mA AC current delivered to the right ventricle endocardium (8). Ventilation was discontinued after the onset of VF. After 8-15 min of untreated VF, CPR, including chest compressions with the LUCAS 2 (PhysioControl Inc, Lund, Sweden) and ventilation with oxygen (tidal volume of $500 \mathrm{~mL}, 10 \mathrm{breaths} / \mathrm{min}$ ), was initiated. More specifically, six pigs had a no-flow time of $8 \mathrm{~min}(8)$, two of $10 \mathrm{~min}$, five of $12 \mathrm{~min}$, three of $13 \mathrm{~min}$, one of $14 \mathrm{~min}$, and five of $15 \mathrm{~min}$. For homogeneous comparison, animals were assigned to three groups of no-flow duration: "short" 8-10 $\min (\mathrm{n}=8)$; "intermediate" $12-13 \min (\mathrm{n}=8)$; and "long" 14-15 $(\mathrm{n}=6)$. After $5 \mathrm{~min}$ of CPR, defibrillation was attempted with a single biphasic 150-J shock, using an MRx defibrillator (Philips Medical Systems, Andover, Mass). If resuscitation was not achieved, CPR was resumed and continued for $1 \mathrm{~min}$ before a subsequent defibrillation. Adrenaline $(30 \mu \mathrm{g} / \mathrm{kg})$ was administered via the right atrium after 2 and $7 \mathrm{~min}$ of CPR. The resuscitation procedures were continued until resuscitation or for a maximum of $15 \mathrm{~min}$. Successful resuscitation was defined as restoration of an organized cardiac rhythm with a mean arterial pressure (MAP) of more than $60 \mathrm{mmHg}$. After that, if VF reoccurred, it was treated by immediate defibrillation. During CPR, the LAD occlusion balloon was deflated to avoid possible rupture during mechanical chest compression, but the catheter was left in place to maintain a partial occlusion of the LAD, which was approximately $75 \%$ of the internal lumen, as previously reported (17). Immediately after resuscitation, the LAD occlusion balloon was reinflated and the catheter correct placement was reconfirmed by fluoroscopy. After successful resuscitation, anesthesia was maintained, and animals were monitored during the 4-h treatment. Forty-five minutes after resuscitation, the LAD catheter was withdrawn. Temperature of the animals was maintained at $38 \pm 0.5^{\circ} \mathrm{C}$ during the whole experiment. After $4 \mathrm{~h}$ of treatment, catheters were removed, wounds were repaired, and the animals were extubated and returned to their cages. Analgesia with butorphanol $(0.1 \mathrm{mg} / \mathrm{kg})$ was administered by intramuscular injection. At the end of the postresuscitation observation period, up to $96 \mathrm{~h}$, animals were reanesthetized for echocardiographic examination and blood sample withdrawn. Animals were then sacrificed painlessly with an intravenous injection of $150 \mathrm{mg} / \mathrm{kg}$ sodium thiopental, and heart and brain were harvested. Autopsy was performed routinely for potential injuries due to $\mathrm{CPR}$ or obfuscating disease.

\section{Measurements}

Hemodynamics, $\mathrm{EtCO}_{2}$, and electrocardiogram were recorded continuously on a personal computer-based acquisition system (WinDaq DATAQ Instruments Inc, Akron, Ohio). The coronary perfusion pressure was computed from the differences in time-coincident diastolic aortic pressure and right atrial pressure. $\mathrm{CO}$ was measured by thermodilution technique (COM-2; Baxter International Inc, Deerfield, Ill). Echocardiography was performed using a phase-array multifrequency $2.5-$ to $5-\mathrm{MHz}$ probe (CX50, Philips, The Netherlands), and left ventricular (LV) ejection fraction (EF) was calculated from single plane two-dimensional echocardiography from apical four chamber view using the modified single-plane Simpson's rule (8). Arterial blood gases were assessed with i-STAT System (Abbott Laboratories, Princeton, NJ). Plasma high-sensitivity cardiac troponin T (hs-cTnT) and serum neuron-specific enolase (NSE) were measured with electrochemiluminescence assays (Roche Diagnostics Italia, Monza, Italy).

As previously described (8), neurologic recovery was assessed with the neurologic alertness score (NAS), ranging from 100 (normal) to 0 (brain death), and with the swine neurologic deficit score (NDS), ranging from 0 (normal) and 400 (brain death). Finally, the functional recovery was evaluated before sacrifice according to overall performance categories (OPCs) as follows: $1=$ normal, $2=$ slight disability, $3=$ severe disability, $4=$ coma, and $5=$ brain brain death or death. Outcome was defined poor when OPC was $\geq 3$. Scores were assessed by veterinarian doctors blinded to no-flow duration.

At sacrifice, the brains were carefully removed from the skulls and fixed in $4 \%$ buffered formalin. Standardized 5-mm coronal slices were taken. The hippocampal CA1 sector and the cortex were chosen as regions of interest and were paraffin embedded. More specifically, the hippocampal CA1 sector was selected because it is well recognized to be associated with postresuscitation cognitive dysfunction in humans and animals $(3,8,18,19)$. The cortex was selected to evaluate the effect of prolonged no-flow durations on cortical neurons, known to be more resistant to ischemia $(18,19)$; and have a histological correspondence with the neurological performances evaluated through the NAS and NDS scores, which are mainly related to the cortical responses. Five-micrometer-thick sections were then obtained and stained with hematoxylin-eosin. The proportion of neuronal loss and degeneration/necrosis (shrunken neurons with deeply acidophilic cytoplasm and pyknotic nucleus) was quantified as absent (0), rare (1), few (2), and numerous (3). An experienced pathologist, blinded to treatment, performed the assessments. For transmission electron microscopy analysis, samples from hippocampus were reduced and fixed with $4 \%$ paraformaldehyde and $2 \%$ glutaraldehyde in phosphate buffer $0.12 \mathrm{~mol} / \mathrm{L} \mathrm{pH} 7.4$ overnight at $4{ }^{\circ} \mathrm{C}$, followed by incubation at room temperature for $2 \mathrm{~h}$ in $2 \% \mathrm{OsO}_{4}$. After dehydration in a graded series of ethanol preparations, tissue samples were cleared in propylene oxide, embedded in epoxy medium (Epoxy Embedding Medium kit; Sigma-Aldrich) and polymerized at $60^{\circ} \mathrm{C}$ for $72 \mathrm{~h}$. Ultra-thin $(60 \mathrm{~nm}$ thick $)$ sections of areas of interest were obtained with a Leica EM UC6 ultramicrotome (Leica Microsystems), counterstained with uranyl acetate and lead citrate and examined with 
SHOCK MONTH 2017

AQ9

TABLE 1. Resuscitation outcomes, hemodynamics, and blood gas analysis

\begin{tabular}{|c|c|c|c|c|}
\hline & Short, $8-10 \mathrm{~min}, \mathrm{n}=8$ & Intermediate, $12-13 \mathrm{~min}, \mathrm{n}=8$ & Long, $14-15 \min , \mathrm{n}=6$ & $P$ value ANOVA \\
\hline Total defibrillations, $\mathrm{n}$ & $3[1 ; 10]$ & $10[2 ; 11]$ & $15[11 ; 26]$ & 0.043 \\
\hline Duration of CPR, s & $315[300 ; 509]$ & $361[325 ; 455]$ & $480[334 ; 757]$ & 0.307 \\
\hline Successful resuscitation, n/n (\%) & $8 / 8(100)$ & $8 / 8(100)$ & $5 / 6(83)$ & 0.247 \\
\hline Long-term survival, n/n (\%) & $7 / 8(88)$ & $5 / 8(63)$ & $1 / 5(20)^{\star}$ & 0.078 \\
\hline \multicolumn{5}{|l|}{ Heart rate, beat/min } \\
\hline BL & $99 \pm 10$ & $93 \pm 8$ & $86 \pm 8$ & 0.602 \\
\hline PR $2 \mathrm{~h}$ & $127 \pm 6$ & $156 \pm 13$ & $164 \pm 11^{*}$ & 0.040 \\
\hline PR $4 \mathrm{~h}$ & $130 \pm 8$ & $129 \pm 12$ & $143 \pm 5$ & 0.595 \\
\hline \multicolumn{5}{|l|}{ Mean arterial pressure, $\mathrm{mmHg}$} \\
\hline BL & $99 \pm 4$ & $101 \pm 3$ & $96 \pm 5$ & 0.702 \\
\hline $\mathrm{PR} 2 \mathrm{~h}$ & $82 \pm 6$ & $85 \pm 6$ & $92 \pm 2$ & 0.571 \\
\hline PR $4 \mathrm{~h}$ & $92 \pm 7$ & $76 \pm 2$ & $78 \pm 6$ & 0.107 \\
\hline \multicolumn{5}{|l|}{ Right atrial pressure, $\mathrm{mmHg}$} \\
\hline $\mathrm{BL}$ & $5 \pm 1$ & $6 \pm 1$ & $8 \pm 1$ & 0.081 \\
\hline PR $2 \mathrm{~h}$ & $7 \pm 1$ & $8 \pm 1$ & $9 \pm 1$ & 0.346 \\
\hline PR $4 \mathrm{~h}$ & $7 \pm 1$ & $7 \pm 0$ & $12 \pm 0^{\star, * \star}$ & $<0.001$ \\
\hline \multicolumn{5}{|l|}{ End-tidal $\mathrm{CO}_{2}, \mathrm{mmHg}$} \\
\hline $\mathrm{BL}$ & $36 \pm 0$ & $37 \pm 1$ & $36 \pm 0$ & 0.406 \\
\hline PR $2 \mathrm{~h}$ & $36 \pm 0$ & $36 \pm 0$ & $37 \pm 1$ & 0.302 \\
\hline $\mathrm{PR} 4 \mathrm{~h}$ & $36 \pm 1$ & $36 \pm 0$ & $37 \pm 0$ & 0.633 \\
\hline \multicolumn{5}{|l|}{ LV CO, L/min } \\
\hline $\mathrm{BL}$ & $4.5 \pm 0.5$ & $4.4 \pm 0.3$ & $4.0 \pm 0.3$ & 0.735 \\
\hline PR $2 \mathrm{~h}$ & $3.3 \pm 0.3$ & $3.3 \pm 0.3$ & $2.8 \pm 0.6$ & 0.604 \\
\hline PR $4 \mathrm{~h}$ & $3.2 \pm 0.3$ & $3.2 \pm 0.4$ & $1.9 \pm 0.1$ & 0.032 \\
\hline LV infarct size area, \% & $9.7 \pm 2.0$ & $6.5 \pm 0.7$ & 13.1 & $\dagger$ \\
\hline \multicolumn{5}{|l|}{$\mathrm{pH}$} \\
\hline $\mathrm{BL}$ & $7.495 \pm 0.017$ & $7.519 \pm 0.016$ & $7.513 \pm 0.027$ & 0.636 \\
\hline PR $2 \mathrm{~h}$ & $7.434 \pm 0.026$ & $7.394 \pm 0.042$ & $7.395 \pm 0.017$ & 0.583 \\
\hline $\mathrm{PR} 4 \mathrm{~h}$ & $7.462 \pm 0.017$ & $7.429 \pm 0.027$ & $7.410 \pm 0.011$ & 0.251 \\
\hline \multicolumn{5}{|l|}{$\mathrm{PaO}_{2}, \mathrm{mmHg}$} \\
\hline $\mathrm{BL}$ & $79 \pm 11$ & $83 \pm 5$ & $83 \pm 2$ & 0.931 \\
\hline PR $2 \mathrm{~h}$ & $89 \pm 7$ & $109 \pm 8$ & $84 \pm 10$ & 0.111 \\
\hline PR $4 \mathrm{~h}$ & $88 \pm 3$ & $100 \pm 11$ & $70 \pm 9$ & 0.074 \\
\hline \multicolumn{5}{|l|}{$\mathrm{PaCO}_{2}, \mathrm{mmHg}$} \\
\hline $\mathrm{BL}$ & $38 \pm 2$ & $37 \pm 1$ & $37 \pm 1$ & 0.789 \\
\hline PR $2 \mathrm{~h}$ & $40 \pm 1$ & $39 \pm 1$ & $41 \pm 1$ & 0.615 \\
\hline $\mathrm{PR} 4 \mathrm{~h}$ & $42 \pm 1$ & $40 \pm 1$ & $42 \pm 1$ & 0.302 \\
\hline \multicolumn{5}{|l|}{$\mathrm{HCO}_{3}, \mathrm{mmol} / \mathrm{L}$} \\
\hline $\mathrm{BL}$ & $29.3 \pm 1.0$ & $30.3 \pm 1.2$ & $29.8 \pm 1.2$ & 0.828 \\
\hline PR $2 \mathrm{~h}$ & $27.2 \pm 0.9$ & $24.5 \pm 2.0$ & $25.4 \pm 0.7$ & 0.353 \\
\hline PR $4 \mathrm{~h}$ & $29.9 \pm 1.1$ & $26.8 \pm 1.3$ & $26.7 \pm 0.4$ & 0.088 \\
\hline \multicolumn{5}{|l|}{$\mathrm{BE}, \mathrm{mmol} / \mathrm{L}$} \\
\hline $\mathrm{BL}$ & $6.4 \pm 1.1$ & $7.4 \pm 1.5$ & $6.7 \pm 1.6$ & 0.865 \\
\hline PR $2 \mathrm{~h}$ & $3.1 \pm 1.4$ & $-0.2 \pm 2.6$ & $0.5 \pm 1.0$ & 0.402 \\
\hline PR $4 \mathrm{~h}$ & $6.1 \pm 1.3$ & $2.5 \pm 1.5$ & $2.0 \pm 0.4$ & 0.089 \\
\hline
\end{tabular}

${ }^{\dagger}$ ANOVA was not performed due to a single animal survived in the "long" group.

${ }^{*} P<0.017$ vs. "short"; ${ }^{* *} P<0.017$ vs. "intermediate."

$\mathrm{BE}$ indicates base excess; $\mathrm{BL}$, baseline; $\mathrm{CO}$, cardiac output; $\mathrm{CPR}$, cardiopulmonary resuscitation; $\mathrm{LV}$, left ventricle; $\mathrm{PaO}_{2}$, arterial partial pressure of oxygen; $\mathrm{PaCO}_{2}$, arterial partial pressure of carbon dioxide; $\mathrm{PR}$, postresuscitation.

Data are expressed as mean \pm SEM.

Energy Filter Transmission Electron Microscope (EFTEM, ZEISS LIBRA 120) equipped with YAG scintillator slow scan CCD camera.

Myocardial infarct was assessed by tetrazolium chloride (TTC) staining. The LV was sliced into 5-mm-thick transverse sections, which were incubated $(20 \mathrm{~min})$ in a solution of TTC and then transferred to $4 \%$ formalin overnight before image analysis. Infarct size was reported as percentage of TTC-negative area relative to LV area (8).

\section{Statistical analysis}

One sample Kolmogorov-Smirnov $Z$ test was used to confirm normal distribution of the data. For comparisons of time-based variables, repeated measures analysis of variance (ANOVA) was used. For comparisons between groups at the given time points, one-way ANOVA with Tukey's multiple comparison was used for normally distributed variables, while Kruskal-Wallis test with Dunn's multiple comparison was used for not normally distributed variables. When the dependent variable was categorical, chi-square test was performed. Linear correlations between parametric variables were calculated using the Pearson correlation coefficient. Spearman test was performed for the nonparametric variable correlation analyses. For survival analysis, Kaplan-Meier survival curves and log-rank (Mantel-Cox) test were used. Data are expressed as mean \pm SEM, except for CPR duration, number of defibrillations delivered, hscTnT and NSE, presented as median [Q1-Q3]. A $P \leq 0.05$ was regarded as statistically significant; for the multiple comparisons, the $P$ value for statistical significance was adjusted to $\leq 0.017$. Data analyses were performed using GraphPad Prism (version 6.05 for Windows; GraphPad Software, La Jolla, Calif).

\section{RESULTS}

No significant differences in hemodynamic parameters, $\mathrm{EtCO}_{2}$, cardiac function, and blood gases analysis were observed among groups at baseline (Table 1). Duration of CPR was similar in the three groups ( $P$ not significant) and all animals were successfully 


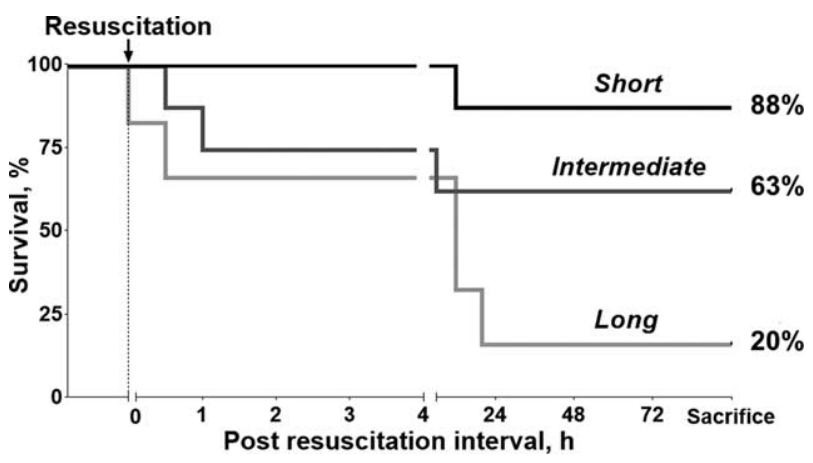

FIG. 1. Kaplan-Meier of postresuscitation survival after an untreated cardiac arrest of short, intermediate, and long duration.

resuscitated except for one animal in the long duration group. More than $80 \%$ of animals in the short and $60 \%$ in the intermediate no-flow duration survived up to 4 days, in contrast to only $20 \%$ in the long duration group $(P=0.017$ long vs. short; Table 1 and Fig. 1). The longer was the duration of no-flow, the higher was the total number of defibrillations delivered ( $r=0.497, P=0.02)$, with a 5-fold difference between the long and the short duration groups (Table 1 and Fig. 2).

Resuscitation outcome, postresuscitation hemodynamics, and blood gas analyses data are shown in Table 1. No significant differences in MAP were observed among the groups, except for a trend toward a higher MAP in the short no-flow group compared to the others (Table 1). Animals subjected to longer durations of no-flow presented significantly higher postresuscitation HR and RAP and a lower LV CO, compared to those in the shorter durations $(P=0.040, P<0.001$, and $P=0.032$, respectively; Table 1$)$.
LV EF decreased in each group after resuscitation (Fig. 2). Postresuscitation LV EF was significantly lower in the longer duration groups compared to the shorter one $(P<0.05)$. LV EF at $4 \mathrm{~h}$ postresuscitation was inversely correlated with the duration of no-flow ( $r=-0.65, P=0.006)$. As expected, an inverse relationship between LV EF and the number of delivered defibrillations was observed $(r=-0.52, P=0.038)$.

LV infarct size was comparable after short or intermediate duration of no-flow, while it was greater in the single animal that survived in the long duration group (Table 1). There was a trend toward higher plasma levels of hs-cTnT in the long duration group (Fig. 2). Hs-cTnT release significantly correlated with the LV infarct size $(r=0.77, P=0.004)$, but not with the duration of no-flow $(r=0.225, P=0.37)$. Nevertheless, levels of hs-cTnT were significantly related to the number of defibrillations received by the animals $(r=0.679, P=0.002)$.

Sixty-two percent of the animals resuscitated in the short and $50 \%$ in the intermediate no-flow duration group survived with a good neurological recovery (OPC $\leq 2$ ), in contrast to no animals in the long duration group, in which only one of the five resuscitated animals survived and had an OPC of three $(P=0.043$, Fig. 3). NAS and NDS showed a trend toward a worse recovery for longer durations of no-flow, with the worst scores observed in the group with the longest duration of untreated CA (Fig. 3). NSE release paralleled the trend observed in the neurological recovery, with higher circulating levels in the instance of longer durations of no-flow $(P<0.001$, Fig. 2). Indeed, the duration of untreated CA strongly correlated with NSE release $(r=0.892, P<0.001)$.

Neurological recovery and NSE data were confirmed by the histological findings, showing greater neuronal degeneration/
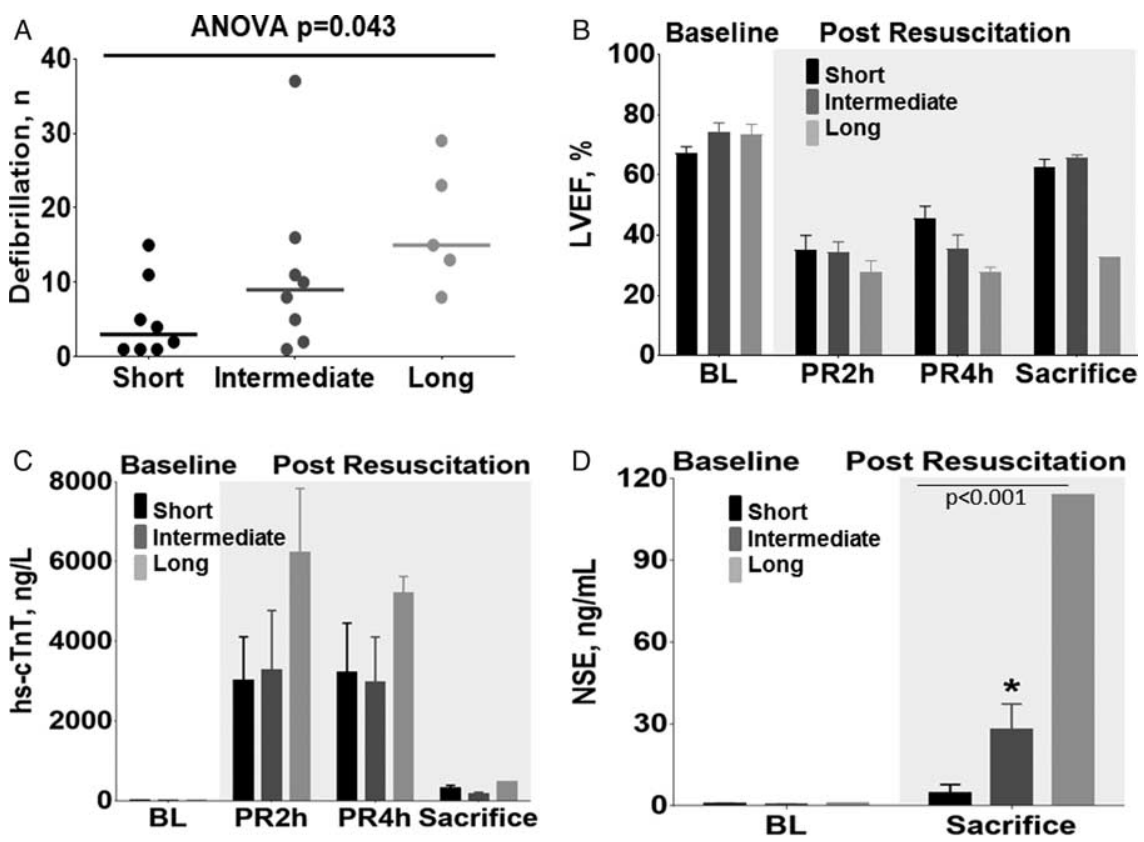

FIG. 2. (A) Total number of defibrillations before successful resuscitation after an untreated cardiac arrest of short, intermediate, and long duration. (B) Left ventricular ejection fraction (LV EF) at baseline (BL), and at 2 and $4 \mathrm{~h}$ postresuscitation (PR), and at sacrifice, after anuntreated cardiac arrest of short, intermediate, and long duration. (C) Plasma high sensitive cardiac troponin T (hs-cTnT) levels at BL, and at 2 and $4 \mathrm{~h}$ PR, and at sacrifice, after an untreated group. 
SHOCK MONTH 2017
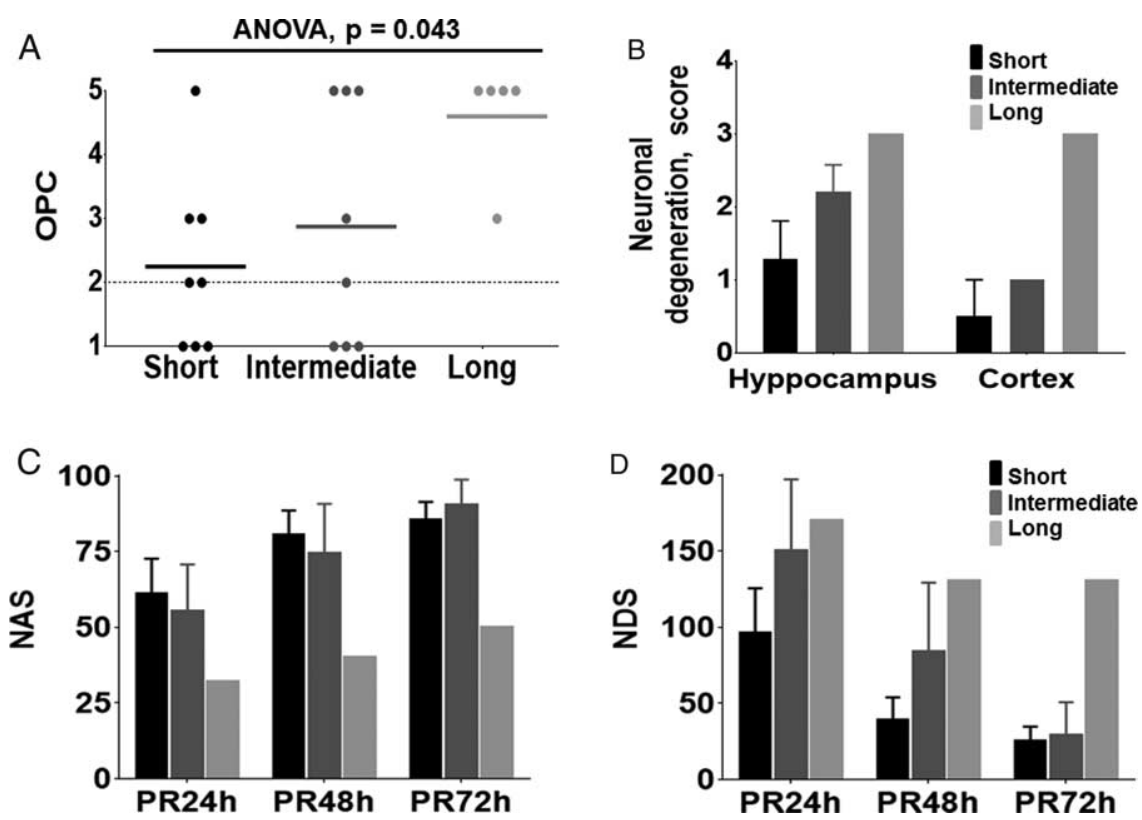

FIG. 3. (A) Overall performance category (OPC); (B) neuronal degeneration in the hippocampal CA1 sector and in cortex, (C) neurological alertness score (NAS); and (D) neuronal deficit score (NDS), after an untreated cardiac arrest of short, intermediate, and long duration.

necrosis in hippocampus and in cortex for increasing no-flow durations, with the single animal survived in the long duration group showing the most severe neuronal injury with associated gliosis and perivascular inflammation (Figs. 3 and 4). Ultrastructural imaging showed mitochondria in neurons undergoing degenerative processes of increasing severity, that is, disruption of cristae and reduction of matrix density, for longer no-flow durations; neuropil, normally composed by a tightly packed myriad of axons, dendrites, and glial processes, also presented a progressive vacuolization and loss of structure (Fig. 5).

\section{DISCUSSION}

The present study systematically investigated, for the first time, the effect of different no-flow intervals on PCAS severity in an experimental model, describing a linear association
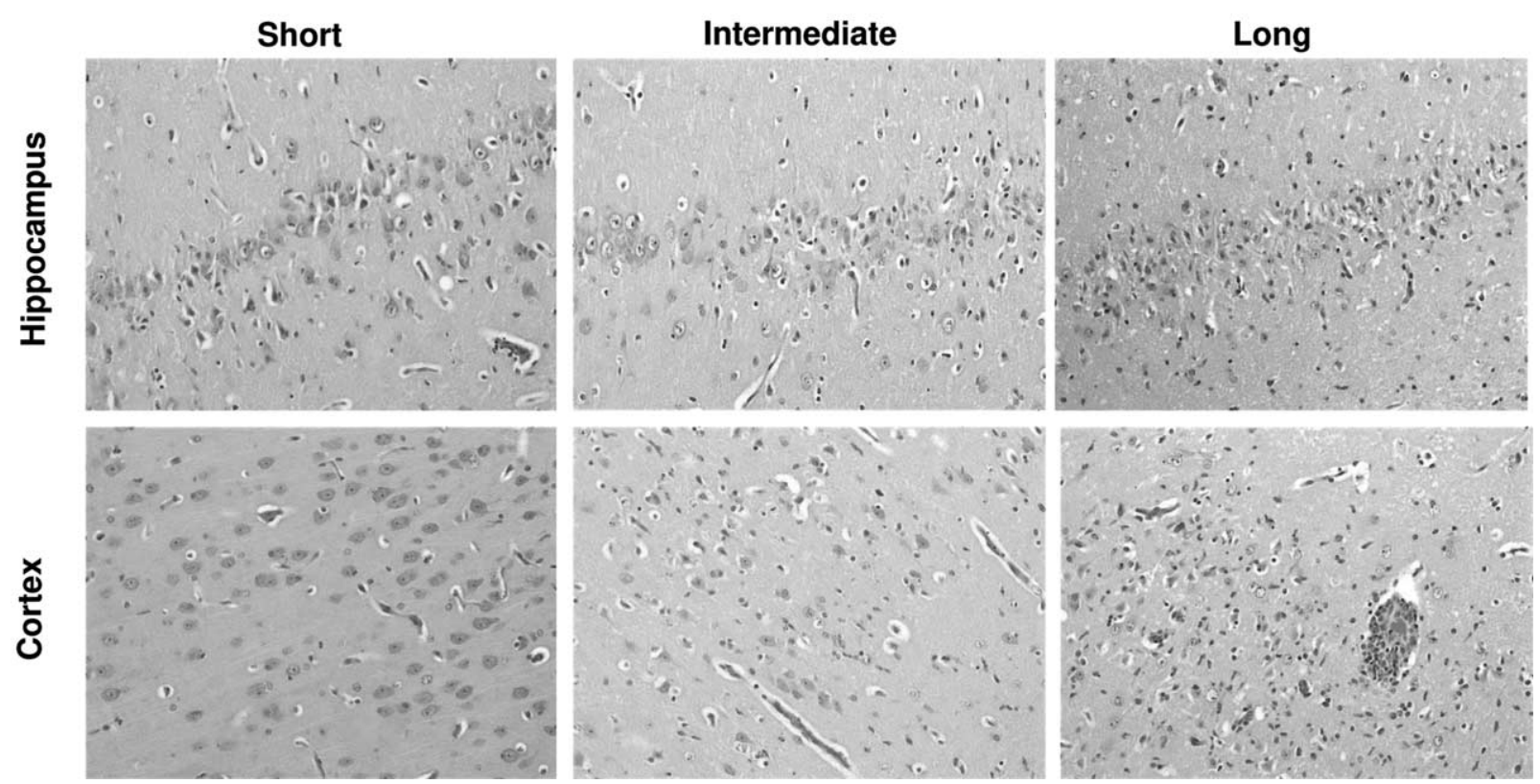

FIG. 4. Hematoxylin-eosin staining $200 \times$ magnification of hippocampal CA1 sector and cortex after an untreated cardiac arrest (CA) of short, intermediate, and long duration. Short duration: hippocampus-some degenerated/necrotic neurons characterized by shrinkage, deeply acidophilic cytoplasm and pyknotic nucleus are intermixed with numerous normal neurons; cortex —normal histological appearance of the nervous tissue. Intermediate duration: hippocampus - numerous degenerated/necrotic neurons with some normal neurons still detectable (on the left side of the picture); cortex-presence of some degenerated/necrotic neurons. Long duration: hippocampus-almost all neurons are degenerated/necrotic; cortex-severe damage characterized by the presence of numerous degenerated/necrotic neurons, gliosis, and perivascular inflammatory cells cuffing. 

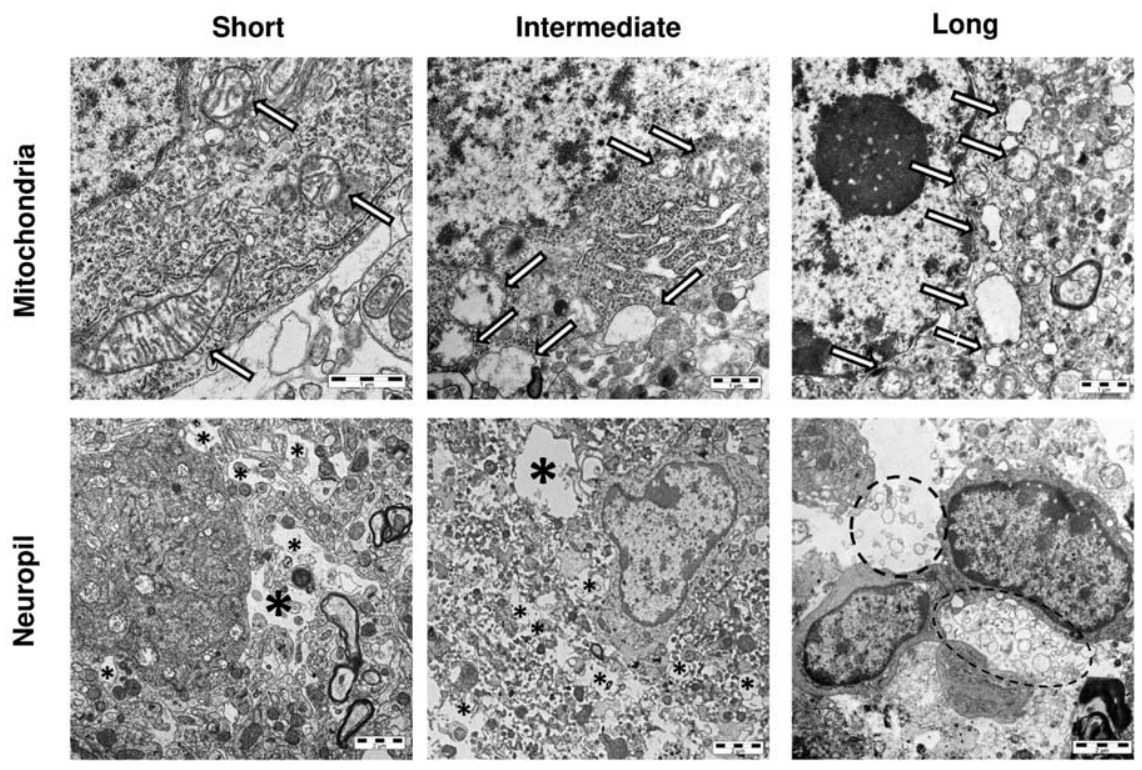

FIG. 5. Ultrastructural analysis of hippocampus. Representative images by transmission electron microscopy of neurons (mitochondria indicated by white arrows) and neuropil (single vacuoles indicated by asterisks and agglomerated vacuoles encircled), after an untreated cardiac arrest (CA) of short, intermediate, and long duration. Short duration: mitochondria show only irregular shapes and moderate damage of inner membranes; neuropil normally composed by a tightly packed myriad of axons, dendrites, and glial processes presents some vacuolated and enlarged processes. Intermediate duration: mitochondria show disruption of cristae and remarkable reduction of matrix density; neuropil shows a more evident vacuolization. Long duration: mitochondria show a complete loss of internal AQ8 components; the confluence of vacuoles determines an edematous-like massive loss of the neuropil.

between the duration of untreated CA and the ensuing heart and brain injury. Indeed, longer no-flow durations were associated with lower percentage of survival and worse postresuscitation myocardial dysfunction and neurological recovery compared to shorter durations. However, the aim of the study was not to describe worse outcomes for longer no-flow durations, but rather to provide evidence and results that can be applied experimentally to design more clinically relevant studies. Showing increasing organ damage and lower survival for longer untreated CA durations was only the pragmatic way to deliver our message.

Duration of no-flow is one of the key features that deeply impact on CA survival and neurological injury (15, 16, 20-22). Designing realistic models for preclinical research, with specific attention to the duration of untreated CA, is therefore crucial to correctly evaluate the potential benefit of new treatments. For this reason, the no-flow duration should be wisely selected to obtain an experimental model that closely reflects the human pathophysiology of CA and PCAS and whose observations might be easily translated clinically (12). CA duration is, however, extremely variable in CPR models (7-11). In a review including 42 experimental articles, significant differences in research methodology have been reported, highlighting a poor clinical representativeness for numerous studies. More specifically, the duration of untreated CA ranged from 0 to $15 \mathrm{~min}$, with the majority of the experiments employing a duration as short as $3 \mathrm{~min}$ or even lesser (12). In other recent reviews on animal models of CPR, longer no-flow intervals, close to $8 \mathrm{~min}$, were reported $(23,24)$. Nevertheless, our study showed that an untreated CA with a duration up to 10 min caused only a limited injury, which accounted for a survival in almost $90 \%$ of resuscitated animals. Only when the duration of no-flow exceeded $12 \mathrm{~min}$, data on survival were similar to those reported clinically (25).

Indeed, in our model, a duration of untreated CA of $12-13$ min showed the best compromise in terms of survival and severity of myocardial and neurological injury, with outcomes that resembled those observed in the clinical scenario, that is, a long-term survival with favorable neurological outcome (OPC 1-2) in 50\% of resuscitated animals $(4,6)$. In contrast, for longer no-flow durations, that is, $14-15 \mathrm{~min}, 0 \%$ of animals survived with a good neurological recovery. Thus, considering clinical survival with good functional recovery, in our opinion an interval of 12-13 min may be an optimal choice for the duration of untreated CA to be used in experimental models, while with longer no-flow intervals, the mortality was unacceptably high. Moreover, in view of using a preclinical model to investigate new neuroprotective interventions, a CA duration $\geq 14$ min determines probably a too extensive neuronal damage with important loss of ultrastructure, such that any potential benefit might be obfuscated.

The myocardial dysfunction observed after $\mathrm{CA}$ is a transient phenomenon due to cell stunning rather than permanent injury that usually spontaneously reverses within the following 2-3 days $(3,22,26)$. In our model, the postresuscitation myocardial dysfunction, evaluated by LV EF, was deeply depressed in all groups of animals during the first $4 \mathrm{~h}$ postresuscitation, with a greater impairment after a duration $\geq 14 \mathrm{~min}$. The worse myocardial dysfunction after a longer untreated CA may be explained as the result of a more severe ischemic insult, supported by the 2 -fold greater infarct size in the single animal that survived and by the higher plasma hs-cTnTs in this group of animals. Another explanation may be represented by the greater number of defibrillations delivered to the animals (27). 


\section{SHOCK MONTH 2017}

Indeed, repetitive defibrillations are known to increase the severity of postresuscitation myocardial dysfunction (28), and in our study, the longer was the duration of no-flow, the greater was the number of defibrillation attempts and such a number of electrical countershocks was significantly related with the systolic dysfunction, represented by the LV EF impairment, and with the myocardial injury, reported as hscTnT release.

We recognize several limitations in the interpretation of our findings. First, the study was not a randomized trial, but included data from an earlier investigation (8), using the same model, and data from new investigations, in which animals were prospectively subjected to progressively longer durations of no-flow. However, the studies were performed consecutively, and investigators, methods for induction of CA and CPR procedures, and methodologies for measurements were the same throughout the experiments, such to allow for reproducibility, consistency of the results, and thus strength of the final message. Second, catheter-related thrombosis might develop very easily during the 45 min postresuscitation LAD occlusion because of the hypercoagulative feature of the porcine blood, potentially leading to emboli release during the LAD catheter withdraw. We decided not to administer heparin during the catheter removal to avoid potential impact on reperfusion; however, all the animals were subjected to the same procedure, limiting the effect of unexpected emboli on differences in outcome among groups. Third, the study did not assess the effect of different CPR or "low-flow" durations, which are known to potentially play a role in outcome (29). Fourth, the effect of body temperature on neurological outcomes was not evaluated, although target temperature management (TTM) is currently a standard of care after OHCA for its beneficial effects on survival and neuroprotection $(4,30,31)$. Nevertheless, the quality of CPR was standardized and the duration of CPR was similar in all the groups. Moreover, all the animals received the same postresuscitation management, with the core temperature maintained at normothermia during the $4 \mathrm{~h}$ of intensive observation. Indeed, the main focus of the study was to assess the impact of the sole no-flow duration on outcome of CA, excluding any other potential bias, that is, TTM or duration of CPR. Therefore, our results are robust to show the direct impact of no-flow duration on the severity of PCAS and outcome of CA. Finally, we acknowledge that results from animal models of CA and CPR may be dependent on the local conditions and skills present in different laboratories. However, this article highlights the need for a standardization of procedures and may pave the route for a share of methodological details and adoption of experimental designs based on the clinical scenario.

The above limitations notwithstanding, in this porcine model, a linear relation between the duration of untreated $\mathrm{CA}$ and the severity of PCAS was observed. Indeed, longer no-flow durations caused greater postresuscitation myocardial and neurological dysfunction and reduced the survival rate, in comparison to shorter durations. A duration of untreated CA of 12-13 min may be an optimal choice for clinically relevant CA animal models because it yields survival rates and neurological outcomes similar to those observed in the clinical scenario.
No-Flow Duration and Cardiac Arrest Models

\section{ACKNOWLEDGMENTS}

The authors thank Physio-Control for the LUCAS 2 compressor and Philips Medical Systems for the MRx defibrillator.

\section{REFERENCES}

1. Monsieurs KG, Nolan JP, Bossaert LL, Greif R, Maconochie IK, Nikolaou NI, Perkins GD, Soar J, Truhlár A, Wyllie J, et al.: European Resuscitation Council Guidelines for Resuscitation 2015: Section 1. Executive summary. Resuscitation 95:1-80, 2015.

2. Gräsner JT, Lefering R, Koster RW, Masterson S, Böttiger BW, Herlitz J, Wnent J, Tjelmeland IB, Ortiz FR, Maurer H, et al.: EuReCa ONE-27 Nations, ONE Europe, ONE Registry: a prospective one month analysis of out-of-hospital cardiac arrest outcomes in 27 countries in Europe. Resuscitation 105:188-195, 2016.

3. Nolan JP, Neumar RW, Adrie C, Aibiki M, Berg RA, Böttiger BW, Callaway C, Clark RS, Geocadin RG, Jauch EC, et al.: Post-cardiac arrest syndrome: epidemiology, pathophysiology, treatment, and prognostication. A Scientific Statement from the International Liaison Committee on Resuscitation; the American Heart Association Emergency Cardiovascular Care Committee; the Council on Cardiovascular Surgery and Anesthesia; the Council on Cardiopulmonary, Perioperative, and Critical Care; the Council on Clinical Cardiology; the Council on Stroke. Resuscitation 79(3):350-379, 2008.

4. Nielsen N, Wetterslev J, Cronberg T, Erlinge D, Gasche Y, Hassager C, Horn J, Hovdenes J, Kjaergaard J, Kuiper M, et al.: Targeted temperature management at $33^{\circ} \mathrm{C}$ versus $36^{\circ} \mathrm{C}$ after cardiac arrest. N Engl J Med 369(23):2197-2206, 2013.

5. Cronberg T, Lilja G, Horn J, Kjaergaard J, Wise MP, Pellis T, Hovdenes J, Gasche Y, Aneman A, Stammet P, et al.: Neurologic function and health-related quality of life in patients following targeted temperature management at $33^{\circ} \mathrm{C}$ vs $36^{\circ} \mathrm{C}$ after out-of-hospital cardiac arrest: a randomized clinical trial. JAMA Neurol 72(6):634-641, 2015.

6. Nolan JP, Soar J, Cariou A, Cronberg T, Moulaert VR, Deakin CD, Bottiger BW, Friberg H, Sunde K, Sandroni C: European Resuscitation Council and European Society of Intensive Care Medicine Guidelines for Post-resuscitation Care 2015: Section 5 of the European Resuscitation Council Guidelines for Resuscitation 2015. Resuscitation 95:202-222, 2015.

7. Ristagno G, Tang W, Huang L, Fymat A, Chang YT, Sun S, Castillo C, Weil MH: Epinephrine reduces cerebral perfusion during cardiopulmonary resuscitation. Crit Care Med 37(4):1408-1415, 2009.

8. Ristagno G, Fumagalli F, Russo I, Tantillo S, Zani DD, Locatelli V, De Maglie M, Novelli D, Staszewsky L, Vago T, et al.: Postresuscitation treatment with argon improves early neurological recovery in a porcine model of cardiac arrest. Shock 41(1):72-78, 2014.

9. Bergan HA, Halvorsen PS, Skulstad H, Edvardsen T, Fosse E, Bugge JF: Successful ECMO-cardiopulmonary resuscitation with the associated postarrest cardiac dysfunction as demonstrated by MRI. Intensive Care Med Exp 3(1):61, 2015.

10. Derwall M, Westerkamp M, Löwer C, Deike-Glindemann J, Schnorrenberger NK, Coburn M, Nolte KW, Gaisa N, Weis J, Siepmann K, et al.: Hydrogen sulfide does not increase resuscitability in a porcine model of prolonged cardiac arrest. Shock 34(2):190-195, 2010.

11. Ji XF, Yang L, Zhang MY, Li CS, Wang S, Cong LH: Shen-fu injection attenuates postresuscitation myocardial dysfunction in a porcine model of cardiac arrest. Shock 35(5):530-536, 2011.

12. Idris AH, Becker LB, Wenzel V, Fuerst RS, Gravenstein N: Lack of uniform definitions and reporting in laboratory models of cardiac arrest: a review of the literature and a proposal for guidelines. Ann Emerg Med 23(1):9-16, 1994.

13. Rea TD, Fahrenbruch C, Culley L, Donohoe RT, Hambly C, Innes J, Bloomingdale M, Subido C, Romines S, Eisenberg MS: CPR with chest compression alone or with rescue breathing. N Engl J Med 363(5):423-433, 2010.

14. Nichol G, Leroux B, Wang H, Callaway CW, Sopko G, Weisfeldt M, Stiell I, Morrison LJ, Aufderheide TP, Cheskes S, et al.: Trial of continuous or interrupted chest compressions during CPR. N Engl J Med 373(23):2203-2214, 2015.

15. Hasselqvist-Ax I, Riva G, Herlitz J, Hollenberg J, Nordberg P, Ringh M, Jonsson M, Axelsson C, Lindqvist J, Karlsson T, et al.: Early cardiopulmonary resuscitation in out-of-hospital cardiac arrest. N Engl J Med 372(24):2307-2315, 2015.

16. Dankiewicz J, Friberg H, Bělohlávek J, Walden A, Hassager C, Cronberg T, Erlinge D, Gasche Y, Hovdenes J, Horn J, et al.: Time to start of cardiopulmonary resuscitation and the effect of target temperature management at $33^{\circ} \mathrm{C}$ and $36^{\circ}$ C. Resuscitation 99:44-49, 2016.

17. Ristagno G, Tang W, Xu TY, Sun S, Weil MH: Outcomes of CPR in the presence of partial occlusion of left anterior descending coronary artery. Resuscitation 75:357-365, 2007.

18. Radovsky A, Safar P, Sterz F, Leonov Y, Reich H, Kuboyama K: Regional prevalence and distribution of ischemic neurons in dog brains 96 hours after cardiac arrest of 0 to 20 minutes. Stroke 26(11):2127-2133, 1995. 
8 SHOCK Vol. $\mathrm{xx}$, No. $\mathrm{x}$

19. Björklund E, Lindberg E, Rundgren M, Cronberg T, Friberg H, Englund E: Ischaemic brain damage after cardiac arrest and induced hypothermia-a systematic description of selective eosinophilic neuronal death. A neuropathologic study of 23 patients. Resuscitation 85(4):527-532, 2014.

20. Sasson C, Rogers MAM, Dahl J, Kellermann AL: Predictors of survival from out-of-hospital cardiac arrest: a systematic review and meta-analysis. Circ Cardiovasc Oual Outcomes 3(1):63-81, 2010.

21. Weaver WD, Cobb LA, Hallstrom AP, Fahrenbruch C, Copass MK, Ray R: Factors influencing survival after out-of-hospital cardiac arrest. J Am Coll Cardiol 7(4):752-757, 1986.

22. Chalkias A, Xanthos T: Post-cardiac arrest brain injury: pathophysiology and treatment. J Neurol Sci 315(1-2):1-8, 2012.

23. Cherry BH, Nguyen AQ, Hollrah RA, Olivencia-Yurvati AH, Mallet RT: Modeling cardiac arrest and resuscitation in the domestic pig. World J Crit Care Med 4(1):1-12, 2015.

24. Vognsen M, Fabian-Jessing BK, Secher N, Løfgren B, Dezfulian C, Andersen LW, Granfeldt A: Contemporary animal models of cardiac arrest: a systematic review. Resuscitation 113:115-123, 2017.

25. Ristagno G, Mauri T, Cesana G, Li Y, Finzi A, Fumagalli F, Rossi G, Grieco N, Migliori M, Andreassi A, et al.: Amplitude spectrum area to guide defibrillation: a validation on 1617 patients with ventricular fibrillation. Circulation 131(5):478-487, 2015
26. Janiczek JA, Winger DG, Coppler P, Sabedra AR, Murray H, Pinsky MR, Rittenberger JC, Reynolds JC, Dezfulian C: Hemodynamic resuscitation characteristics associated with improved survival and shock resolution after cardiac arrest. Shock 45(6):613-619, 2016.

27. Ristagno G, Wang T, Tang W, Sun S, Castillo C, Weil MH: High-energy defibrillation impairs myocyte contractility and intracellular calcium dynamics. Crit Care Med 36(11 Suppl.):S422-S427, 2008.

28. Tang W, Snyder D, Wang J, Huang L, Chang YT, Sun S, Weil MH: One-shock versus three-shock defibrillation protocol significantly improves outcome in a porcine model of prolonged ventricular fibrillation cardiac arrest. Circulation 113(23):2683-2689, 2006.

29. Reynolds JC, Grunau BE, Rittenberger JC, Sawyer KN, Kurz MC, Callaway $\mathrm{CW}$ : Association between duration of resuscitation and favorable outcome after out-of-hospital cardiac arrest: implications for prolonging or terminating resuscitation. Circulation 134(25):2084-2094, 2016.

30. Bernard SA, Gray TW, Buist MD, Jones BM, Silvester W, Gutteridge G, Smith K: Treatment of comatose survivors of out-of-hospital cardiac arrest with induced hypothermia. $N$ Engl $J$ Med 346(8): 557-563, 2002.

31. Hypothermia after Cardiac Arrest Study Group. Mild therapeutic hypothermia to improve the neurologic outcome after cardiac arrest. $N$ Engl $\mathrm{J} \mathrm{Med}$ 346(8):549-556, 2002.
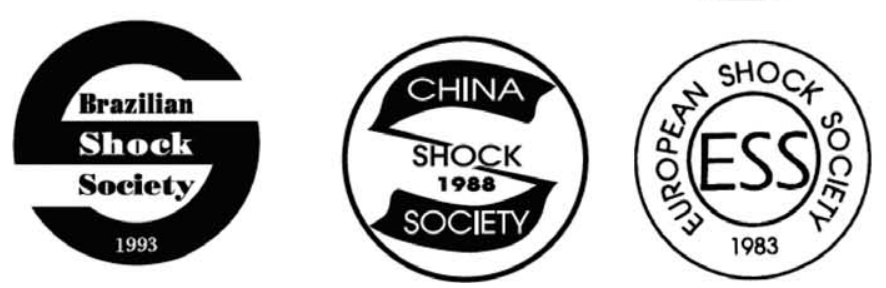
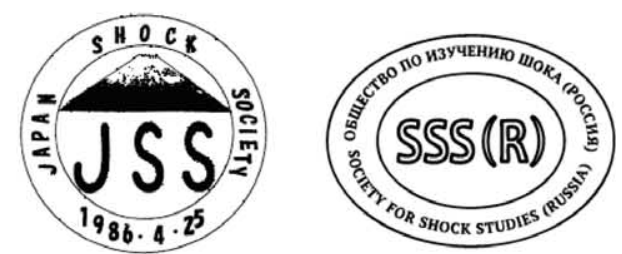


\section{SHK}

Manuscript No. -D-17-00221

\section{SHOCK}

Dear Author,

During the preparation of your manuscript for typesetting, some queries have arisen. These are listed below. Please check your typeset proof carefully and mark any corrections in the margin as neatly as possible or compile them as a separate list. This form should then be returned with your marked proof/list of corrections to the Production Editor.

\section{QUERIES: to be answered by AUTHOR}

\begin{tabular}{|l|l|}
\hline QUERY NO. & \multicolumn{1}{c|}{ QUERY DETAILS } \\
\hline$<$ AQ1 & $\begin{array}{l}\text { Please confirm whether surnames/family } \\
\text { names (red) have been identified } \\
\text { correctly in the author byline. }\end{array}$ \\
Affiliations have been set as per style. \\
Please check for accuracy of \\
information.
\end{tabular}

